OPEN ACCESS

Edited by:

Tetsuo Kida

National Institute for Physiological Sciences (NIPS), Japan

Reviewed by: Jennifer Strafford Stevens, Emory University School of Medicine,

USA

Brian Edward Engdahl,

University of Minnesota, USA

${ }^{*}$ Correspondence: Amy S. Badura-Brack abadura@creighton.edu

Received: 10 February 2017 Accepted: 07 April 2017 Published: 25 April 2017

Citation: Badura-Brack AS, Heinrichs-Graham E, McDermott TJ, Becker KM, Ryan TJ, Khanna MM and Wilson TW (2017) Resting-State Neurophysiological Abnormalities in Posttraumatic Stress Disorder: A Magnetoencephalography Study.

Front. Hum. Neurosci. 11:205. doi: 10.3389/fnhum.2017.00205

\section{Resting-State Neurophysiological Abnormalities in Posttraumatic Stress Disorder: A Magnetoencephalography Study}

\author{
Amy S. Badura-Brack ${ }^{1 *}$, Elizabeth Heinrichs-Graham ${ }^{2,3}$, Timothy J. McDermott H,2, $^{1,2}$ \\ Katherine M. Becker ${ }^{2,4}$, Tara J. Ryan ${ }^{1,5}$, Maya M. Khanna ${ }^{1}$ and Tony W. Wilson ${ }^{2,3}$ \\ ${ }^{1}$ Department of Psychology, Creighton University, Omaha, NE, USA, ${ }^{2}$ Center for Magnetoencephalography (MEG), University \\ of Nebraska Medical Center (UNMC), Omaha, NE, USA, ${ }^{3}$ Department of Neurological Sciences, University of Nebraska \\ Medical Center (UNMC), Omaha, NE, USA, ${ }^{4}$ Department of Psychology, Colorado State University, Fort Collins, CO, USA, \\ ${ }^{5}$ Department of Psychology, Simon Fraser University, Burnaby, BC, Canada
}

Posttraumatic stress disorder (PTSD) is a debilitating psychiatric condition that is common in veterans returning from combat operations. While the symptoms of PTSD have been extensively characterized, the neural mechanisms that underlie PTSD are only vaguely understood. In this study, we examined the neurophysiology of PTSD using magnetoencephalography (MEG) in a sample of veterans with and without PTSD. Our primary hypothesis was that veterans with PTSD would exhibit aberrant activity across multiple brain networks, especially those involving medial temporal and frontal regions. To this end, we examined a total of 51 USA combat veterans with a battery of clinical interviews and tests. Thirty-one of the combat veterans met diagnostic criteria for PTSD and the remaining 20 did not have PTSD. All participants then underwent high-density MEG during an eyes-closed resting-state task, and the resulting data were analyzed using a Bayesian image reconstruction method. Our results indicated that veterans with PTSD had significantly stronger neural activity in prefrontal, sensorimotor and temporal areas compared to those without PTSD. Veterans with PTSD also exhibited significantly stronger activity in the bilateral amygdalae, parahippocampal and hippocampal regions. Conversely, healthy veterans had stronger neural activity in the bilateral occipital cortices relative to veterans with PTSD. In conclusion, these data suggest that veterans with PTSD exhibit aberrant neural activation in multiple cortical areas, as well as medial temporal structures implicated in affective processing.

Keywords: PTSD, MEG, resting-state, posttraumatic stress disorder, magnetoencephalography (MEG)

\section{INTRODUCTION}

Posttraumatic stress disorder (PTSD) is a psychiatric disorder involving re-experiencing, avoidance, negative mood and cognition and arousal symptoms (American Psychiatric Association, 2013). The prevalence of PTSD in veterans of Operation Enduring Freedom (OEF) and Operation Iraqi Freedom (OIF) is estimated at 23\% (Bagalman, 2013), which is about 2.5 times higher than the $8.9 \%$ USA lifetime prevalence rate of PTSD (American Psychiatric Association, 2013). Greater combat exposure is associated with more severe PTSD symptomatology, as well as greater psychiatric 
comorbidity in veterans (Dedert et al., 2009; Vasterling et al., 2010; Bagalman, 2013) consistent with the inter-relatedness of the core constructs of PTSD, anxiety and depression (Byllesby et al., 2016).

Previous fMRI and PET studies of PTSD have consistently reported aberrant activation in the anterior, middle and posterior cingulate cortices, medial prefrontal and middle frontal gyri, as well as the insula and medial temporal structures such as the hippocampus and amygdalae of patients with PTSD relative to matched controls (Rabinak et al., 2011; Morey et al., 2012; Pitman et al., 2012; Sripada et al., 2012; Boccia et al., 2016). Meta-analysis has suggested that the anterior cingulate cortices (ACC) and bilateral amygdala may be the most commonly hyper-activated regions in patients with PTSD (Hayes et al., 2012). Structural studies have also reported reduced volume in similar regions, including the hippocampal (O'Doherty et al., 2015) and ACC in patients with PTSD relative to controls (Meng et al., 2014; O'Doherty et al., 2015). Beyond the classic fronto-limbic regions, studies have reported abnormalities in multiple cortical areas including parietal regions, default-mode network areas, dorsal prefrontal regions, motor regions and occipital areas in those with PTSD compared to demographically-matched controls (Eckart et al., 2011; Schuff et al., 2011; Liu et al., 2012; Mueller-Pfeiffer et al., 2013). Thus, it seems unlikely that the disorder can be uniquely attributed to dysfunction in the fronto-limbic regions that have been historically implicated.

As with other areas of psychiatric and neurologic research, resting-state fMRI studies of PTSD have increased and several fMRI studies have reported altered resting-state functional connectivity in participants with PTSD compared to controls, especially in regions of the default-mode network (Qin et al., 2012; Zhou et al., 2012; Du et al., 2015; Reuveni et al., 2016). In addition, resting-state studies using magnetoencephalography (MEG) have appeared and these studies have suggested hyperconnectivity in medial temporal areas of patients with PTSD (Dunkley et al., 2014, 2015), as well as miscommunication between right temporo-parietal cortices and the rest of the brain (Engdahl et al., 2010). All of these studies focused on connectivity and those by Dunkley et al. (2014, 2015) used a mask that limited their analysis to specific a priori fronto-limbic areas. Thus, whether the regions themselves were dysfunctional (i.e., not just connectivity), and whether aberrant processing would have been detected beyond frontolimbic regions in patients with PTSD remains unknown. One preliminary MEG study of traumatic memory recall in women with PTSD found activation largely outside of fronto-limbic regions (Cottraux et al., 2015), calling attention to other brain regions. In the current study, we examined a group of combat veterans with and without PTSD using resting-state MEG and clinical assessments. We focused on resting-state activity as it circumvents possible differences in behavioral performance from biasing the neural signals, and used MEG due to its excellent spatiotemporal precision and close relationship with the underlying neurophysiology. The two groups of veterans were closely-matched on important demographic variables, and following MEG their data were source imaged and evaluated using a voxel-by-voxel whole brain approach. Our primary goal was to identify brain regions with altered spontaneous neuronal activity in combat veterans with PTSD. Our central hypothesis was that veterans with PTSD would exhibit aberrant restingstate (i.e., spontaneous) activity in multiple cortical, medial temporal and fronto-limbic areas compared to veterans without PTSD.

\section{MATERIALS AND METHODS}

We recruited a community sample of 51 USA military veterans living near Omaha, NE, USA, who had experienced combat in conflicts in Iraq and Afghanistan (between 2003 and 2014) using television commercials, flyers, and social media. Following clinical assessment (see below), it was determined that 31 of these male combat veterans met the diagnostic criteria for PTSD and the remaining 20 male combat veterans did not meet criteria for PTSD or any other psychiatric or neurological condition. Participants without PTSD were matched to those with PTSD on age, education, ethnicity and handedness. General exclusionary criteria included any medical diagnosis affecting CNS function, known brain neoplasm or lesion, history of significant head injury and ferromagnetic implants. This study was carried out in accordance with the recommendations of the Creighton University Institutional Review Board with written informed consent from all subjects. All subjects gave written informed consent in accordance with the Declaration of Helsinki. The protocol was approved by the Creighton University Institutional Review Board.

\section{Psychological Assessment}

PTSD was diagnosed using the Clinician Administered PTSD Scale (CAPS; Blake et al., 1995) and the symptom Frequency of 1, symptom Intensity of 2 rule (Weathers et al., 1999). Veterans in the healthy control group did not meet criteria for PTSD or any other psychiatric disorder. Participants were assessed for comorbid diagnoses using the Mini International Neuropsychiatric Interview (MINI; Sheehan et al., 1998), and none of the participants had a comorbid diagnosis of psychosis, bipolar disorder, obsessive-compulsive disorder, or current substance dependence. All participants also completed a battery of psychological questionnaires including the Patient Health Questionnaire (PHQ-9), which measured depression (Kroenke et al., 2001); the Toronto Alexithymia Scale (TAS-20), which measured difficulty identifying and describing feelings (Bagby et al., 1994); the State-Trait Anxiety Inventory (STAI), which measured both state and trait anxiety (Spielberger et al., 1983); the Deployment Risk and Resilience Inventory (DRRI), which measured combat exposure (Vogt et al., 2008); and the Life Events Checklist (LEC) measured traumatic events across the lifespan (Blake et al., 1995). Finally, as noted above, all veterans also completed the CAPS (Blake et al., 1995), which is the gold standard diagnostic interview for PTSD. The CAPS produced a PTSD symptom severity score for each participant (including those without PTSD), which we report in the results. 


\section{MEG Data Acquisition and sMRI Coregistration}

All recordings were conducted in a one-layer MSR with active shielding engaged. With an acquisition bandwidth of $0.1-330 \mathrm{~Hz}$, neuromagnetic responses were sampled continuously at $1 \mathrm{kHz}$ using an Elekta system with 306 magnetic sensors, including 204 planar gradiometers and 102 magnetometers (Elekta, Helsinki, Finland). MEG data from each participant were individually corrected for head motion and subjected to noise reduction using the signal space separation method with a temporal extension (tSSS; Taulu and Simola, 2006).

Prior to MEG measurement, four coils were attached to the participant's head and the locations of these coils, together with the three fiducial points and scalp surface, were determined with a 3-D digitizer (Fastrak 3SF0002, Polhemus Navigator Sciences, Colchester, VT, USA). Once the participant was positioned for MEG recording, an electric current with a unique frequency label (e.g., $322 \mathrm{~Hz}$ ) was fed to each of the coils. This induced a measurable magnetic field and allowed each coil to be localized in reference to the sensors throughout the recording session. Since coil locations were also known in head coordinates, all MEG measurements could be transformed into a common coordinate system. With this coordinate system (including the scalp surface points), each participant's MEG data were coregistered with T1-weighted sMRI data using the Statistical Parametric Mapping software (SPM12; Litvak et al., 2011) implemented in Matlab (Mathworks, Inc.).

\section{MEG Preprocessing, Source Imaging and Statistics}

The continuous 6-min magnetic time series was divided into epochs of $4096 \mathrm{~ms}$ duration, and epochs with artifacts were identified using an automated procedure that identified flat and high amplitude segments for rejection; this procedure was supplemented with visual inspection and ultimately resulted in less than $5 \%$ of epochs being rejected. Artifact-free epochs were filtered $1-54 \mathrm{~Hz}$ and then downsampled to $180 \mathrm{~Hz}$ prior to image reconstruction to reduce computational burden. The downsampled data across all 306 sensors were then imaged using the Bayesian multiple sparse priors (MSP) approach implemented in the SPM12 software (Litvak et al., 2011; Wellcome Trust Centre for Neuroimaging).

In our MSP approach, source space consisted of 8196 dipolar voxels equally-distributed throughout gray matter. Data covariance was calculated across the whole epoch from $1 \mathrm{~Hz}$ to $54 \mathrm{~Hz}$. Prior to inversion, the MEG sensor data were transformed into a set of orthogonal modes using a singular value decomposition over the lead field matrix, and a temporal projector was applied to reduce the data to 16 temporal modes (Friston et al., 2008; Henson et al., 2009; López et al., 2014). A Variational Laplace approach was then used to estimate the combination of hyper-parameters that maximized free energy in the Bayesian framework. Model selection used both automatic relevance determination and greedy search schemes, and the covariance matrices produced by each and the sensor noise covariances were mixed using Variational Laplace in a second inversion step. The resulting single covariance matrix was used to get the posterior mean and variance of the current density, with output images written at $2 \mathrm{~mm}^{3}$ resolution. See López et al. (2014) for a detailed description of the MSP approach to Bayesian source reconstruction.

The resulting 3D maps of functional brain activity were statistically evaluated in SPM12 using a mass univariate approach based on the general linear model. Briefly, the effects of group were examined using a mixed random effects model, whereas one-sample $t$-tests were conducted to probe task effects in each group. Statistical maps for task effects were thresholded at $p<0.001$; whereas group effect maps were thresholded at $p<0.01$. All statistical maps were adjusted for multiple comparisons using a spatial extent threshold (cluster restriction), which was calculated directly from the data according to the theory of Gaussian random fields. All statistical analyses for behavioral and clinical variables were conducted in SPSS (Release 21.0.0).

\section{RESULTS}

\section{Participant Demographics and Clinical Measures}

No participants were receiving psychotherapy at the time of enrollment. Eleven veterans with PTSD were on stable (no change for at least 6 months) doses of psychiatric medication (4 SSRIs, 3 benzodiazepines and 4 mood stabilizers), and the other 20 veterans with PTSD were not taking any psychiatric medication. One veteran in the non-PTSD group was taking a SSRI. Mean age was 32.13 (SD: 5.98 ) years in the PTSD group and 31.60 (SD: 7.18$)$ years in the control group $(p=0.78)$. Mean educational level was 14.71 (SD: 2.41 ) years in the PTSD group and 14.13 (SD: 2.70 ) years in the control group $(p=0.42)$. By design, veterans with PTSD had significantly higher PTSD scores than the no PTSD comparison group $(p<0.0001)$. As expected, veterans with PTSD also reported higher rates of depressive and anxious symptoms compared to those without PTSD ( $p$ 's $<0.01$;

\begin{tabular}{lll}
\hline TABLE 1 | Psychological assessment scores. & \\
\hline Measure (symptom) & Group & Mean (SD) \\
\hline CAPS (PTSD)** & PTSD & $72.03(15.31)$ \\
& Non-PTSD & $22.95(11.94)$ \\
PHQ-9 (depression)** & PTSD & $11.77(6.14)$ \\
TAS-20 (alexithymia)** & Non-PTSD & $2.35(2.28)$ \\
& PTSD & $57.77(13.57)$ \\
STAI Y1 (state anxiety)** & Non-PTSD & $42.40(10.50)$ \\
& PTSD & $45.29(9.85)$ \\
STAI Y2 (trait anxiety)** & Non-PTSD & $35.90(5.90)$ \\
& PTSD & $47.65(12.21)$ \\
DRRI (combat exposure)* & Non-PTSD & $31.20(6.94)$ \\
& PTSD & $38.07(17.21)$ \\
& Non-PTSD & $26.59(19.11)$ \\
\hline
\end{tabular}

PTSD $(n=31)$; Non-PTSD $(n=20) .{ }^{*}$ Scores significantly different between groups at $p<0.01$. ${ }^{*}$ Scores significantly different between groups at $p<0.05$. 
see Table 1). Such increased depressive and anxious symptoms are commonly associated with PTSD and are part of the typical clinical presentation of PTSD. Lastly, veterans with PTSD had significantly more severe combat exposure relative to those without PTSD ( $p<0.05$; Table 1) according to the DRRI, but the two groups did not statistically differ in exposure to lifelong trauma exposure including childhood and/or civilian trauma experiences.

\section{MEG Data: Resting-State Effects}

We evaluated the brain areas active in veterans with and without PTSD during the resting-state prior to examining group differences. In both groups, significant neuronal activity was observed in bilateral areas of the occipital, inferior parietal, posterior cingulate and sensorimotor cortices $(p<0.001$, corrected). Significant activity was also detected in bilateral medial temporal structures, including amygdalae, hippocampi and parahippocampal regions of each group $(p<0.001$, corrected). In veterans with PTSD, bilateral prefrontal activity was also found $(p<0.001$, corrected; Figure 1).

\section{MEG Data: PTSD Group Comparisons}

Veterans with PTSD exhibited significantly stronger neural activity in bilateral regions of the precentral and postcentral gyri, paracentral lobule, middle temporal gyri, inferior temporal sulci, DLPFC and the right superior temporal sulcus compared to veterans without PTSD ( $p<0.01$, corrected; Figure 2$)$. Veterans with PTSD also had significantly stronger activity in the bilateral amygdala, parahippocampal and hippocampal regions relative to those without PTSD ( $p<0.01$, corrected). In contrast, veterans without PTSD had stronger activity in the left and right lateral occipital cortices compared to veterans with PTSD $(p<0.01$, corrected; Figure 2).

In addition, we performed exploratory analyses to identify the primary frequency of neural activity underlying our critical findings, and as a sanity check to ensure high-frequency responses were not missed due to the broadband approach of our primary analysis (i.e., low-frequency responses are generally stronger than high-frequency and can be missed using such approaches). To this end, we filtered MEG signals into conventional frequency bands (e.g., theta, alpha) and imaged each band separately. Our results indicated that theta $(4-7 \mathrm{~Hz})$ was the critical frequency for our medial temporal findings (e.g., amygdala, parahippocampal, hippocampal), while alpha $(8-14 \mathrm{~Hz})$ activity was central to our occipital, temporal and sensorimotor findings. Our findings in the DLPFC extended across theta and alpha (i.e., group differences were observed in the DLPFC for both bands). Beta and gamma differences were much weaker, non-significant and likely made a negligible contribution to our primary findings.

\section{DISCUSSION}

We used high-density MEG and advanced image reconstruction methods to quantify resting-state (i.e., spontaneous) activity in combat veterans with and without PTSD. Our main findings were that veterans with PTSD exhibited significantly stronger activity relative those without PTSD in bilateral motor regions, lateral temporal areas, the DLPFC and multiple medial temporal areas including the hippocampi and amygdala. The opposite pattern of stronger activity in the non-PTSD group was observed in the lateral occipital cortices. Major strengths of

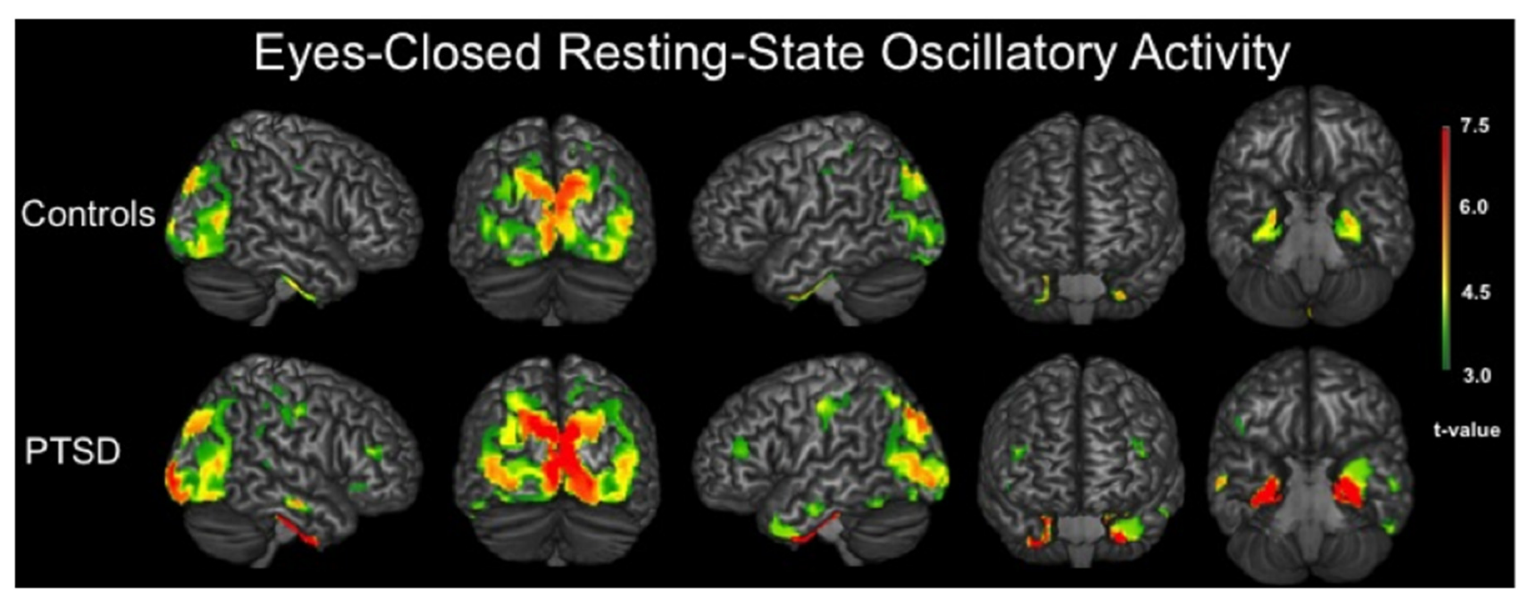

FIGURE 1 | 3D renditions showing brain regions with significant resting-state neuronal activity in combat veterans without posttraumatic stress disorder (PTSD; top row) and with PTSD (bottom row). All group-level maps have been thresholded at ( $p<0.001$, corrected); a color scale bar showing respective $t$-values appears on the far right. Brain regions with significant activity in veterans without PTSD included bilateral occipital regions, inferior parietal cortices, postcentral gyri, posterior cingulate, parahippocampal gyri and other medial temporal areas. For veterans with PTSD, bilateral activity was observed in the primary motor and somatosensory cortices (i.e., precentral and postcentral gyri), superior parietal cortices, inferior parietal cortices, occipital regions, dorsolateral prefrontal cortices, inferior temporal sulci, posterior cingulate and bilateral medial temporal structures including parahippocampal gyri, hippocampi and the amygdala. 


\section{PTSD vs Control Between Group Differences}

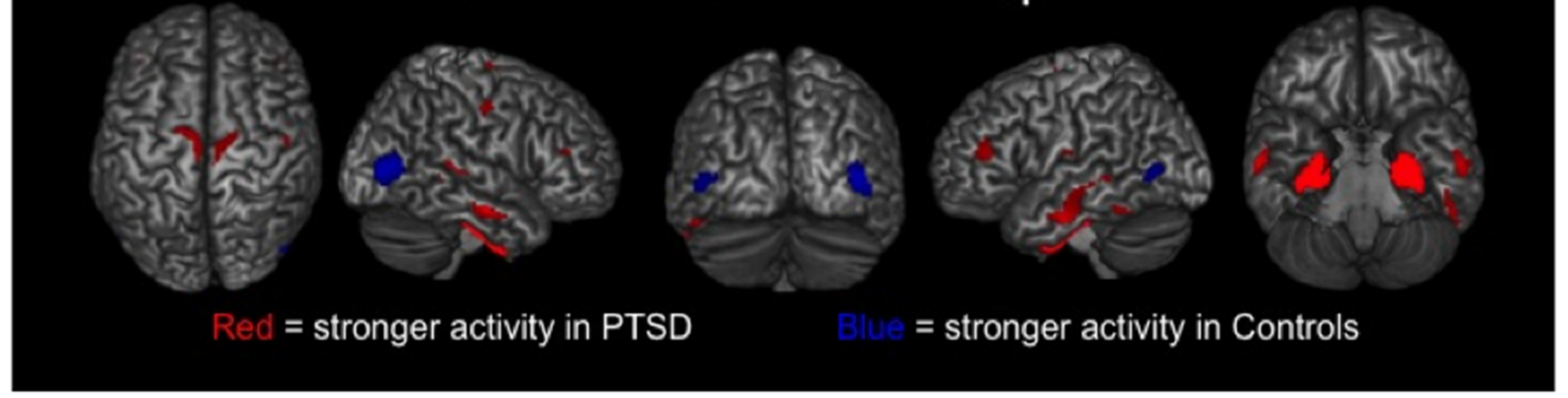

FIGURE 2 | Group differences in resting-state neuronal activity between combat veterans with and without PTSD were found in several brain regions. In the top row, brain areas exhibiting significantly $(p<0.01$, corrected) stronger activity in veterans with PTSD are shown in red, whereas regions with significantly weaker activity in these patients appear in blue. As shown, veterans with PTSD had stronger resting-state neuronal activity in bilateral medial temporal areas (e.g., amygdalae, parahippocampal), sensorimotor, temporal and prefrontal regions, along with significantly weaker activity in left and right lateral occipital cortices.

this study included the thorough clinical assessments, stateof-the-art neurophysiological imaging, and the close matching of veterans with PTSD to a psychiatrically-healthy combatexposed comparison group. Lifetime traumatic events were similar between groups, and although combat exposure was greater in the PTSD group, the comparison sample had also experienced significant combat-related traumas. Given that the experience of trauma in the absence of PTSD symptoms appears to have a lasting effect on the brain (Stark et al., 2015), being able to draw conclusions specifically about the presence of PTSD requires a trauma exposed control group, yet such a control group is often absent in PTSD brain research. Below, we discuss the implications of these findings for understanding the neural circuitry of combat-related PTSD.

In each group, we observed significant resting-state neural activity in bilateral occipital, inferior parietal and sensorimotor cortices, as well as medial temporal structures including bilateral amygdala, hippocampus and parahippocampal cortices. In fact, with the exception of the DLPFC (observed in PTSD group only), both groups exhibited very similar patterns of resting-state neural activity. Group comparisons of the patients with PTSD and matched combat-exposed controls revealed regionally-specific patterns of increased and decreased neuronal activity during the resting-state. Specifically, we found stronger spontaneous neural activity in the bilateral sensorimotor cortices (pre and postcentral gyri), paracentral lobule and DLPFC in veterans with PTSD as compared to non-PTSD controls. The sensorimotor and prefrontal findings are consistent with evidence of aberrant neurodynamics in veterans with PTSD in these same brain regions in two recent MEG task-based studies (Badura-Brack et al., 2015; McDermott et al., 2016), as well as the results of a group classification study (Gong et al., 2014). The latter study indicated that the primary motor and prefrontal cortices were among the most important brain areas for group prediction, along with parietal and occipital regions (Gong et al., 2014). As expected, our PTSD group also had significantly stronger resting-state activity in medial temporal areas known to be critical for threat alert and emotion, including the hippocampi, parahippocampal gyri and amygdalae (Eckart et al., 2011; Morey et al., 2012; Meng et al., 2014). Interestingly, we also observed significantly weaker activity in the left and right lateral occipital cortices in veterans with PTSD compared to those without PTSD, which is congruent with other findings such as reduced occipital gray matter in PTSD (Chao et al., 2012; Li et al., 2014), and the resting-state predictive value of the occipital lobes in PTSD (Gong et al., 2014).

The amygdala, hippocampus and surrounding structures are perhaps the most well-documented brain regions in PTSD (Rabinak et al., 2011; Morey et al., 2012; Pitman et al., 2012; Sripada et al., 2012), and these areas significantly differentiated veterans with and without PTSD in our study. These regions, along with the parahippocampal gyri, are critical memory structures along the ventral visual processing stream, and are central to how traumatic information is stored and processed in PTSD (Brewin et al., 1996, 2010). We also found significant and widespread differences in more dorsal visual processing areas, suggestive of dorsal stream aberrations in PTSD. Influential theories of PTSD symptomatology suggest that re-experiencing (a key symptom) events are derived from visual sensory impressions that were inadequately integrated into autobiographical memory at the time of the trauma (Brewin et al., 1996, 2010; Ehlers and Clark, 2000). Involuntary memory intrusions in PTSD tend to have visual details predominating (Ehlers et al., 2002; Hackmann et al., 2004), and posttraumatic symptoms may be related to priming of these visual memories. Both sensorimotor and occipital areas along the dorsal processing stream appear to differentiate posttraumatic flashbacks from other autobiographical memories (Whalley et al., 2013). Our findings clearly highlight the need for future research into these brain regions, which have been woefully under-examined in the pathophysiology of PTSD. 
In conclusion, we examined the neural basis of PTSD in a sample of USA combat veterans using advanced MEG methods and clinical assessments. We focused on the amplitude of resting-state (i.e., spontaneous) neural activity using a voxel-based whole brain approach, as previous studies had largely restricted their analyses to a priori brain regions and focused exclusively on functional connectivity among regions included in the a priori mask. Our results indicated that veterans with PTSD had stronger resting-state neural activity in bilateral areas of the occipital, inferior parietal, sensorimotor and parahippocampal cortices, as well as the amygdalae and hippocampi. The opposite pattern was observed in lateral occipital cortices bilaterally. In summary, PTSD is associated with aberrations in widespread brain regions, including dorsal neocortical areas and the classic medial temporal structures often linked to affective processing and PTSD symptomatology. Future task-based studies should closely evaluate their data for baseline level differences (i.e., preceding stimulus onset), as our findings suggest that spontaneous neural activity may be altered in PTSD. Future studies should also evaluate women and PTSD resulting from different types of trauma to determine whether the neurophysiological effects differ from what we observed in male combat veterans.

Finally, our study sample was not without limitations, including our focus on men and combat-related PTSD, possible medication or anxiety effects in the veterans with PTSD, and expected differences in the severity of combat exposure between the two groups. In addition, we examined only the resting state using MEG (i.e., no comparison task), and some of our findings in the medial temporal lobe will need to be confirmed by future studies given the reduced sensitivity of MEG to neural sources distant from the sensors. That said, we used both magnetometers and gradiometers for source reconstruction, and magnetometers are inherently more sensitive to neural activity in distant brain

\section{REFERENCES}

American Psychiatric Association. (2013). The Diagnostic and Statistical Manual of Mental Disorders: DSM 5th Edn. Arlington: American Psychiatric Association.

Badura-Brack, A. S., Becker, K. M., McDermott, T. J., Ryan, T. J., Becker, M. M., Hearley, A. R., et al. (2015). Decreased somatosensory activity to non-threatening touch in combat veterans with posttraumatic stress disorder. Psychiatry Res. 233, 194-200. doi: 10.1016/j.pscychresns.2015.06.012

Bagalman, E. (2013). Mental disorders among OEF/OIF veterans using VA health care: facts and figures. Washington. Available online at: https://fas.org/sgp/crs/misc/R41921.pdf

Bagby, R. M., Parker, J. D., and Taylor, G. J. (1994). The twenty-item Toronto Alexithymia Scale-I. Item selection and cross-validation of the factor structure. J. Psychosom Res. 38, 23-32. doi: 10.1016/0022-3999(94) 90005-1

Blake, D. D., Weathers, F. W., Nagy, L. M., Kaloupek, D. G., Gusman, F. D., Charney, D. S., et al. (1995). The development of a clinicianadministered PTSD scale. J. Trauma. Stress 8, 75-90. doi: 10.1007/bf021 05408

Boccia, M., D'Amico, S., Bianchini, F., Marano, A., Giannini, A. M., and Piccardi, L. (2016). Different neural modifications underpin PTSD after different traumatic events: an fMRI meta-analytic study. Brain Imaging Behav. 10, 226-237. doi: 10.1007/s11682-015-9387-3 regions. We also observed anticipated group differences in these medial temporal structures, providing converging evidence on the veracity of these findings, as MEG recordings would be equally sensitive (or insensitive) to such activity across both groups. MEG studies reporting neural activity in deeper brain structures such as the cerebellum, amygdala and other medial temporal regions are becoming more common across the field (Kessler et al., 2006; Dalal et al., 2008; Salvadore et al., 2009, 2010; Wilson et al., 2009, 2010, 2011, 2017; Cornwell et al., 2012a,b, 2014; Muthuraman et al., 2014; McDermott et al., 2016; Proskovec et al., 2016; Pu et al., 2017). In summary, our MEG results converge nicely with well-established findings in these deeper structures in PTSD, draw needed attention to multiple cortical regions including expected prefrontal structures, as well as calling for increased consideration of sensorimotor, temporal and occipital areas in the pathophysiology of PTSD.

\section{AUTHOR CONTRIBUTIONS}

All authors certify having participated sufficiently in the work to take public responsibility for the content, including participation in the concept, design, analysis, writing and/or revision of the manuscript.

\section{FUNDING}

This research was supported by a grant from the nonprofit organization At Ease, USA (AB-B), grant R01-MH103220 from the National Institutes of Health (TWW), and grant \#1539067 from the National Science Foundation (TWW). The funders had no role in study design, data collection and analysis, decision to publish, or preparation of the manuscript.

Brewin, C. R., Dalgleish, T., and Joseph, S. (1996). A dual representation theory of posttraumatic stress disorder. Psychol. Rev. 103, 670-686. doi: 10.1037/0033295x.103.4.670

Brewin, C. R., Gregory, J. D., Lipton, M., and Burgess, N. (2010). Intrusive images in psychological disorders: characteristics, neural mechanisms, and treatment implications. Psychol. Rev. 117, 210-232. doi: 10.1037/a0018113

Byllesby, B. M., Durham, T. A., Forbes, D., Armour, C., and Elhai, J. D. (2016). An investigation of PTSD's core dimensions and relations with anxiety and depression. Psychol. Trauma 8, 214-217. doi: 10.1037/tra0 000081

Chao, L. L., Lenoci, M., and Neylan, T. C. (2012). Effects of post-traumatic stress disorder on occipital lobe function and structure. Neuroreport 23, 412-419. doi: 10.1097/WNR.0b013e328352025e

Cornwell, B. R., Arkin, N., Overstreet, C., Carver, F. W., and Grillon, C. (2012a). Distinct contributions of human hippocampal theta to spatial cognition and anxiety. Hippocampus 22, 1848-1859. doi: 10.1002/hipo.22019

Cornwell, B. R., Salvadore, G., Furey, M., Marquardt, C. A., Brutsche, N. E., Grillon, C., et al. (2012b). Synaptic potentiation is critical for rapid antidepressant response to ketamine in treatment-resistant major depression. Biol. Psychiatry 72, 555-561. doi: 10.1016/j.biopsych.2012. 03.029

Cornwell, B. R., Overstreet, C., and Grillon, C. (2014). Spontaneous fast $\gamma$ activity in the septal hippocampal region correlates with spatial learning in humans. Behav. Brain Res. 261, 258-264. doi: 10.1016/j.bbr.2013.12.031 
Cottraux, J., Lecaignard, F., Yao, S.-N. N., De Mey-Guillard, C., Haour, F., Delpuech, C., et al. (2015). Magneto-encephalographic (MEG) brain recordings during traumatic memory recall in women with post-traumatic stress disorder: a pilot study. Encephale 41, 202-208. doi: 10.1016/j.encep.2014. 03.002

Dalal, S. S., Guggisberg, A. G., Edwards, E., Sekihara, K., Findlay, A. M., Canolty, R. T., et al. (2008). Five-dimensional neuroimaging: localization of the time-frequency dynamics of cortical activity. Neuroimage 40, 1686-1700. doi: 10.1016/j.neuroimage.2008.01.023

Dedert, E. A., Green, K. T., Calhoun, P. S., Yoash-Gantz, R., Taber, K. H., Mumford, M. M., et al. (2009). Association of trauma exposure with psychiatric morbidity in military veterans who have served since September 11, 2001. J. Psychiatr. Res. 43, 830-836. doi: 10.1016/j.jpsychires.2009.01.004

Du, M.-Y. Y., Liao, W., Lui, S., Huang, X.-Q. Q., Li, F., Kuang, W.-H. H., et al. (2015). Altered functional connectivity in the brain default-mode network of earthquake survivors persists after 2 years despite recovery from anxiety symptoms. Soc. Cogn. Affect. Neurosci. 10, 1497-1505. doi: $10.1093 /$ scan/nsv040

Dunkley, B. T., Doesburg, S. M., Jetly, R., Sedge, P. A., Pang, E. W., and Taylor, M. J. (2015). Characterising intra- and inter-intrinsic network synchrony in combat-related post-traumatic stress disorder. Psychiatry Res. 234, 172-181. doi: 10.1016/j.pscychresns.2015.09.002

Dunkley, B. T., Doesburg, S. M., Sedge, P. A., Grodecki, R. J., Shek, P. N., Pang, E. W., et al. (2014). Resting-state hippocampal connectivity correlates with symptom severity in post-traumatic stress disorder. Neuroimage Clin. 5, 377-384. doi: 10.1016/j.nicl.2014.07.017

Eckart, C., Stoppel, C., Kaufmann, J., Tempelmann, C., Hinrichs, H., Elbert, T., et al. (2011). Structural alterations in lateral prefrontal, parietal and posterior midline regions of men with chronic posttraumatic stress disorder. J. Psychiatry Neurosci. 36, 176-186. doi: 10.1503/jpn.100010

Ehlers, A., and Clark, D. M. (2000). A cognitive model of posttraumatic stress disorder. Behav. Res. Ther. 38, 319-345. doi: 10.1016/S0005-7967(99) 00123-0

Ehlers, A., Hackmann, A., Steil, R., Clohessy, S., Wenninger, K., and Winter, H. (2002). The nature of intrusive memories after trauma: the warning signal hypothesis. Behav. Res. Ther. 40, 995-1002. doi: 10.1016/s0005-7967(01) 00077-8

Engdahl, B., Leuthold, A. C., Tan, H.-R. M.-R., Lewis, S. M., Winskowski, A. M., Dikel, T. N., et al. (2010). Post-traumatic stress disorder: a right temporal lobe syndrome? J. Neural Eng. 7:066005. doi: 10.1088/1741-2560/7/6/ 066005

Friston, K., Harrison, L., Daunizeau, J., Kiebel, S., Phillips, C., Trujillo-Barreto, N., et al. (2008). Multiple sparse priors for the M/EEG inverse problem. Neuroimage 39, 1104-1120. doi: 10.1016/j.neuroimage.2007.09.048

Gong, Q., Li, L., Du, M., Pettersson-Yeo, W., Crossley, N., Yang, X., et al. (2014). Quantitative prediction of individual psychopathology in trauma survivors using resting-state FMRI. Neuropsychopharmacology 39, 681-687. doi: 10.1038/npp.2013.251

Hackmann, A., Ehlers, A., Speckens, A., and Clark, D. (2004). Characteristics and content of intrusive memories in PTSD and their changes with treatment. J. Trauma. Stress 17, 231-240. doi: 10.1023/b:jots.0000029266.88369.fd

Hayes, J. P., Hayes, S. M., and Mikedis, A. M. (2012). Quantitative meta-analysis of neural activity in posttraumatic stress disorder. Biol. Mood Anxiety Disord. 2:9. doi: 10.1186/2045-5380-2-9

Henson, R. N., Mattout, J., Phillips, C., and Friston, K. J. (2009). Selecting forward models for MEG source-reconstruction using model-evidence. Neuroimage 46, 168-176. doi: 10.1016/j.neuroimage.2009.01.062

Kessler, K., Biermann-Ruben, K., Jonas, M., Siebner, H. R., Bäumer, T., Münchau, A., et al. (2006). Investigating the human mirror neuron system by means of cortical synchronization during the imitation of biological movements. Neuroimage 33, 227-238. doi: 10.1016/j.neuroimage.2006.06.014

Kroenke, K., Spitzer, R. L., and Williams, J. B. (2001). The PHQ-9: validity of a brief depression severity measure. J. Gen. Intern. Med. 16, 606-613. doi: 10.1046/j.1525-1497.2001.016009606.x

Li, L., Wu, M., Liao, Y., Ouyang, L., Du, M., Lei, D., et al. (2014). Grey matter reduction associated with posttraumatic stress disorder and traumatic stress. Neurosci. Biobehav. Rev. 43, 163-172. doi: 10.1016/j.neubiorev.2014. 04.003
Litvak, V., Mattout, J., Kiebel, S., Phillips, C., Henson, R., Kilner, J., et al. (2011). EEG and MEG data analysis in SPM8. Comput. Intell. Neurosci. 2011: 852961. doi: 10.1155/2011/852961

Liu, Y., Li, Y.-J. J., Luo, E.-P. P., Lu, H.-B. B., and Yin, H. (2012). Cortical thinning in patients with recent onset post-traumatic stress disorder after a single prolonged trauma exposure. PLoS One 7:e39025. doi: 10.1371/journal. pone.0039025

López, J. D., Litvak, V., Espinosa, J. J., Friston, K., and Barnes, G. R. (2014). Algorithmic procedures for Bayesian MEG/EEG source reconstruction in SPM. Neuroimage 84, 476-487. doi: 10.1016/j.neuroimage.2013.09.002

McDermott, T. J., Badura-Brack, A. S., Becker, K. M., Ryan, T. J., Khanna, M. M., Heinrichs-Graham, E., et al. (2016). Male veterans with PTSD exhibit aberrant neural dynamics during working memory processing: an MEG study. J. Psychiatry Neurosci. 41, 251-260. doi: 10.1503/jpn.150058

Meng, Y., Qiu, C., Zhu, H., Lama, S., Lui, S., Gong, Q., et al. (2014). Anatomical deficits in adult posttraumatic stress disorder: a meta-analysis of voxel-based morphometry studies. Behav. Brain Res. 270, 307-315. doi: 10.1016/j.bbr.2014. 05.021

Morey, R. A., Gold, A. L., LaBar, K. S., Beall, S. K., Brown, V. M., Haswell, C. C., et al. (2012). Amygdala volume changes in posttraumatic stress disorder in a large case-controlled veterans group. Arch. Gen. Psychiatry 69, 1169-1178. doi: 10.1001/archgenpsychiatry.2012.50

Mueller-Pfeiffer, C., Schick, M., Schulte-Vels, T., O’Gorman, R., Michels, L., Martin-Soelch, C., et al. (2013). Atypical visual processing in posttraumatic stress disorder. Neuroimage Clin. 3, 531-538. doi: 10.1016/j.nicl.2013. 08.009

Muthuraman, M., Hellriegel, H., Hoogenboom, N., Anwar, A. R., Mideksa, K. G., Krause, H., et al. (2014). Coherent source and connectivity analysis on simultaneously measured EEG and MEG data during isometric contraction. Conf. Proc. IEEE Eng. Med. Biol. Soc. 2014, 6365-6368. doi: 10.1109/EMBC. 2014.6945084

O’Doherty, D. C., Chitty, K. M., Saddiqui, S., Bennett, M. R., and Lagopoulos, J. (2015). A systematic review and meta-analysis of magnetic resonance imaging measurement of structural volumes in posttraumatic stress disorder. Psychiatry Res. 232, 1-33. doi: 10.1016/j.pscychresns.2015. 01.002

Pitman, R. K., Rasmusson, A. M., Koenen, K. C., Shin, L. M., Orr, S. P., Gilbertson, M. W., et al. (2012). Biological studies of post-traumatic stress disorder. Nat. Rev. Neurosci. 13, 769-787. doi: 10.1038/nrn3339

Proskovec, A. L., Heinrichs-Graham, E., and Wilson, T. W. (2016). Aging modulates the oscillatory dynamics underlying successful working memory encoding and maintenance. Hum. Brain Mapp. 37, 2348-2361. doi: $10.1002 / \mathrm{hbm} .23178$

$\mathrm{Pu}$, Y., Cornwell, B. R., Cheyne, D., and Johnson, B. W. (2017). The functional role of human right hippocampal/parahippocampal $\theta$ rhythm in environmental encoding during virtual spatial navigation. Hum. Brain Mapp. 38, 1347-1361. doi: 10.1002/hbm.23458

Qin, L. D., Wang, Z., Sun, Y.-W. W., Wan, J.-Q. Q., Su, S.-S. S., Zhou, Y., et al. (2012). A preliminary study of alterations in default network connectivity in post-traumatic stress disorder patients following recent trauma. Brain Res. 1484, 50-56. doi: 10.1016/j.brainres.2012.09.029

Rabinak, C. A., Angstadt, M., Welsh, R. C., Kenndy, A. E., Lyubkin, M., Martis, B., et al. (2011). Altered amygdala resting-state functional connectivity in post-traumatic stress disorder. Front. Psychiatry 2:62. doi: 10.3389/fpsyt. 2011.00062

Reuveni, I., Bonne, O., Giesser, R., Shragai, T., Lazarovits, G., Isserles, M., et al. (2016). Anatomical and functional connectivity in the default mode network of post-traumatic stress disorder patients after civilian and military-related trauma. Hum. Brain Mapp. 37, 589-599. doi: 10.1002/hbm. 23051

Salvadore, G., Cornwell, B. R., Colon-Rosario, V., Coppola, R., Grillon, C., Zarate, C. A., et al. (2009). Increased anterior cingulate cortical activity in response to fearful faces: a neurophysiological biomarker that predicts rapid antidepressant response to ketamine. Biol. Psychiatry 65, 289-295. doi: 10.1016/j.biopsych.2008.08.014

Salvadore, G., Cornwell, B. R., Sambataro, F., Latov, D., Colon-Rosario, V., Carver, F., et al. (2010). Anterior cingulate desynchronization and functional connectivity with the amygdala during a working memory task predict 
rapid antidepressant response to ketamine. Neuropsychopharmacology 35, 1415-1422. doi: 10.1038/npp.2010.24

Schuff, N., Zhang, Y., Zhan, W., Lenoci, M., Ching, C., Boreta, L., et al. (2011). Patterns of altered cortical perfusion and diminished subcortical integrity in posttraumatic stress disorder: an MRI study. Neuroimage 54, S62-S68. doi: 10.1016/j.neuroimage.2010.05.024

Sheehan, D. V., Lecrubier, Y., Sheehan, K. H., Amorim, P., Janavs, J., Weiller, E., et al. (1998). The mini-international neuropsychiatric interview (M.I.N.I.): the development and validation of a structured diagnostic psychiatric interview for DSM-IV and ICD-10. J. Clin. Psychiatry 59, 22-33;quiz34-57.

Spielberger, C. D., Gorsuch, R. L., Lushene, R., Vagg, P. R., and Jacobs, G. A. (1983). Manual for the State-Trait Anxiety Inventory. Palo Alto, CA: Consulting Psychologists Press.

Sripada, R. K., King, A. P., Garfinkel, S. N., Wang, X., Sripada, C. S., Welsh, R. C., et al. (2012). Altered resting-state amygdala functional connectivity in men with posttraumatic stress disorder. J. Psychiatry Neurosci. 37, 241-249. doi: 10.1503/jpn.110069

Stark, E. A., Parsons, C. E., Van Hartevelt, T. J., Charquero-Ballester, M., McManners, H., Ehlers, A., et al. (2015). Post-traumatic stress influences the brain even in the absence of symptoms: a systematic, quantitative meta-analysis of neuroimaging studies. Neurosci. Biobehav. Rev. 56, 207-221. doi: 10.1016/j. neubiorev.2015.07.007

Taulu, S., and Simola, J. (2006). Spatiotemporal signal space separation method for rejecting nearby interference in MEG measurements. Phys. Med. Biol. 51, 1759-1768. doi: 10.1088/0031-9155/51/7/008

Vasterling, J. J., Proctor, S. P., Friedman, M. J., Hoge, C. W., Heeren, T., King, L. A., et al. (2010). PTSD symptom increases in Iraq-deployed soldiers: comparison with nondeployed soldiers and associations with baseline symptoms, deployment experiences and postdeployment stress. J. Trauma. Stress 23, 41-51. doi: 10.1002/jts.20487

Vogt, D. S., Proctor, S. P., King, D. W., King, L. A., and Vasterling, J. J. (2008). Validation of scales from the deployment risk and resilience inventory in a sample of operation iraqi freedom veterans. Assessment 15, 391-403. doi: $10.1177 / 1073191108316030$

Weathers, F., Ruscio, A., and Keane, T. (1999). Psychometric properties of nine scoring rules for the clinician-administered posttraumatic stress disorder scale. Psychol. Assess. 11, 124-133. doi: 10.1037/1040-3590.11. 2.124

Whalley, M. G., Kroes, M. C., Huntley, Z., Rugg, M. D., Davis, S. W., and Brewin, C. R. (2013). An fMRI investigation of posttraumatic flashbacks. Brain Cogn. 81, 151-159. doi: 10.1016/j.bandc.2012.10.002

Wilson, T. W., Proskovec, A. L., Heinrichs-Graham, E., O’Neill, J., Robertson, K. R., Fox, H. S., et al. (2017). Aberrant neuronal dynamics during working memory operations in the aging HIV-infected brain. Sci. Rep. 7:41568. doi: 10.1038/srep41568

Wilson, T. W., Slason, E., Asherin, R., Kronberg, E., Reite, M. L., Teale, P. D., et al. (2010). An extended motor network generates $\beta$ and $\gamma$ oscillatory perturbations during development. Brain Cogn. 73, 75-84. doi: 10.1016/j.bandc.2010.03.001

Wilson, T. W., Slason, E., Asherin, R., Kronberg, E., Teale, P. D., Reite, M. L., et al. (2011). Abnormal $\gamma$ and $\beta$ MEG activity during finger movements in earlyonset psychosis. Dev. Neuropsychol. 36, 596-613. doi: 10.1080/87565641.2011. 555573

Wilson, T. W., Slason, E., Hernandez, O. O., Asherin, R., Reite, M. L., Teale, P. D., et al. (2009). Aberrant high-frequency desynchronization of cerebellar cortices in early-onset psychosis. Psychiatry Res. 174, 47-56. doi: 10.1016/j.pscychresns. 2009.03.009

Zhou, Y., Wang, Z., Qin, L. D., Wan, J. Q., Sun, Y. W., Su, S. S., et al. (2012). Early altered resting-state functional connectivity predicts the severity of post-traumatic stress disorder symptoms in acutely traumatized subjects. PLoS One 7:e46833. doi: 10.1371/journal.pone.0046833

Conflict of Interest Statement: The authors declare that the research was conducted in the absence of any commercial or financial relationships that could be construed as a potential conflict of interest.

Copyright (C) 2017 Badura-Brack, Heinrichs-Graham, McDermott, Becker, Ryan, Khanna and Wilson. This is an open-access article distributed under the terms of the Creative Commons Attribution License (CC BY). The use, distribution or reproduction in other forums is permitted, provided the original author(s) or licensor are credited and that the original publication in this journal is cited, in accordance with accepted academic practice. No use, distribution or reproduction is permitted which does not comply with these terms. 